Chapter 2

\title{
Graphene Formation on Metal Surfaces Investigated by In-situ STM
}

\author{
Guocai Dong, Dirk W. van Baarle and \\ Joost W. M. Frenken \\ Additional information is available at the end of the chapter \\ http://dx.doi.org/10.5772/56435
}

\section{Introduction}

Graphene, single-layer graphite, has drawn great attention recently, both because of its special properties [1-5], and because of its wide-range potential applications, for example in futuregeneration electronics [4, 6-8]. Chemical vapor deposition (CVD) of hydrocarbons on transition metals (TMs) provides a practical method for graphene production [9-17]. With this chapter we would like to summarize our investigations with in-situ scanning tunneling microscopy (STM), aimed at understanding the growth of graphene on metal surfaces. The 'fruit fly' of our work has been graphene formation on $\mathrm{Rh}(111)$. Observations on this surface are generalized into a simple physical picture that should be suitable to interpret the formation of graphene also on other metals.

The first experimentally produced and verified graphene was obtained in 2004 by mechanical exfoliation of graphite [18]. Soon in 2010 The Nobel Prize in Physics was awarded to Andre Geim and Konstantin Novoselov at the University of Manchester "for groundbreaking experiments regarding the two-dimensional material graphene". Scotch tape was used to repeatedly split graphite crystals into progressively thinner pieces. Many experiments on graphene have been performed using this method [5, 19]. It nicely provides experimentalists with the best quality in topographical flatness [19] and electron mobility [5]. The drawback of this method is, of course, reproducibility. From an application point of view, it is hard to imagine any production facility using microscopes to find tiny bits of graphene for electronic devices.

Since 2004, researchers have tried out many ways for a reproducible, bottom-up production of graphene. One method is to heat silicon carbide to high temperatures $\left(>1100^{\circ} \mathrm{C}\right)$, in order 
to make it sublime silicon and thereby reduce its surface to graphene [20,21]. The choice of the face of the silicon carbide that is used for graphene creation, either silicon-terminated or carbon-terminated, strongly influences the thickness (i.e. the number of layers), mobility, and carrier density of the graphene. This process produces a graphene sample size that is dependent on the size of the $\mathrm{SiC}$ substrate. However, the size of the domains is still in the order of hundreds of nanometers [20,21]. Although the quantum Hall effect (QHE) could be measured with the graphene samples created by this method [20], the electronic mobility in this graphene only reached $2700 \mathrm{~cm}^{2} \mathrm{~V}^{-1} \mathrm{~s}^{-1}$, [22] which is much lower than for the graphene obtained by the scotch tape method.

A very promising alternative method to produce precisely mono-layer of graphene is chemical vapor deposition (CVD) on metal surfaces [9-17, 23-28]. Actually, graphitic layers on metals had been investigated much earlier [11,29-33], before specific attention was paid to graphene. It is the method that we have concentrated on in our work. Metal surfaces as favorable substrates for graphene growth by CVD have catalysts for hydrocarbon decomposition and they strongly bind to the graphene that forms, while newly arriving hydrocarbon molecules do not stick to or decompose on the graphene that has already formed. Also as the solubility of carbon in a metal often depends strongly on temperature, graphene can also be made using segregated carbon $[9,12,16,27,30,34]$. After samples have been prepared at high temperature and cooled down subsequently, it is not easy to distinguish which kind of carbon, segregated or deposited, has been responsible for the formation of the graphene layer.

As a consequence of its strong interaction with the substrate, graphene on top of a metal is very different from the idealized free-standing graphene. It does not have the interesting electronic properties mentioned before, but it does have its own special appeal, for example, being the template for the self assembly of additional overlayers [35], or the graphene-metal contact serving a spin filter [36, 37], etc. In order to produce free-standing graphene, several recipes have been introduced for transferring graphene from metals onto other substrates [15, 34,38 , 39]. With these recipes, CVD-grown graphite layers on metals have become a good candidate for producing high-quality graphene. A promising carrier mobility of 4,000 to 7,350 $\mathrm{cm}^{2} \mathrm{~V}^{-1} \mathrm{~s}^{-1}$ has already been reported for graphene, obtained via this route [40-43]. The size of graphene made by CVD method is reported up to 30 inch [43].

No matter in what form the graphene is produced, free-standing or on a substrate, for many applications it is the structural quality of the graphene that limits its properties and therefore the quality of the final products in which it can be applied. The typical types of imperfections introduced by the CVD method on metals are impurities and structural defects. The problem of impurities can be solved by using a sufficiently clean production setup and sufficiently clean materials (metal substrate and precursor gasses). The structural defects include other carbon phases (e.g. metal carbide), domain boundaries between different graphene patches, point defects [13] (e.g. pentagons and heptagons [44]) and thickness variations (voids or multilayers). It has been demonstrated that such defects can influence the electronic properties of graphene [44]. To reduce and eventually completely avoid the occurrence of structural defects, full control of the formation mechanism of graphene is necessary. This can only come through a thorough, atomic-scale understanding of the process. Once we have acquired a full under- 
standing of the basic mechanisms of graphene formation and possible competing processes, we can use that to subsequently develop an optimal growth recipe.

This chapter will start with a brief introduction of the in-situ STM technique and compare it with other in-situ techniques, e.g. low-energy electron microscopy (LEEM) and scanning electron microscopy (SEM). Ethylene deposition on $\mathrm{Rh}(111)$ is taken as an example to demonstrated the formation of graphene on metal surfaces. This system is ideal to demonstrate the interaction between carbon and metals, as all effects are present 'at the same time': in addition to graphene formation, we observe carbide formation and dissolution of carbon into the substrate, which later re-surfaces and deteriorates the quality of the graphene. A universal physical picture for graphene formation and competing phenomena on metal surfaces will be provided. This picture is based on very general surface-science knowledge, which makes it easy to be adapted to other CVD procedures and to graphene formation on other metals. Solid experimental evidences will be shown to confirm the picture. Guided by this picture, we will demonstrate several paths to improve the quality of graphene formed on a metal surface. Each path shows its own advantages and disadvantages. We hope these demonstrations will help the readers understand the universal picture better and use it to improve graphene quality for different applications.

\section{STM for in-situ measurements}

Direct real-space microscopic observation during the formation of graphene is obviously important for understanding the kinetic processes and thermal dynamics as well as for improving the quality of graphene. However, due to the high growth temperature, to realize such observation one has to face many technical challenges. Firstly, any temperature changes in the macro structures of the microscope will cause dramatic thermal drift on the atomic scale in the imaging process. Secondly, even though one can image at a constant, high temperature, it is more difficult to stabilize the whole system at higher temperatures than for example room temperature or lower.

Many common microscopy techniques, such as STM, LEEM, and SEM, etc. have been performed in situ to investigate the graphene formation at high temperatures on metals $[9,16,38$, 45-47]. In table 1, the working conditions and the performances of these techniques are listed for comparison. With some special designs, all of these methods satisfy the required conditions for graphene growth, i.e., sample temperature and gas pressure. Although there are arguments about the definition of the resolution, high-temperature STM is superior in spatial resolution. In fact, it is the only technique which can provide images in which the moiré pattern between graphene and metal substrate (about $3 \mathrm{~nm}$ ) can be clearly distinguished even at the growth condition of graphene [46]. The higher resolution certainly provides more information for understanding the growth kinetics of graphene. On the other hand, LEEM and SEM can image much larger areas, which gives a macroscopic knowledge of the growth. In STM, the measurement itself has less influence the formation, because the energy of the tunneling electrons is typically not more a few $\mathrm{eV}$ instead of $\mathrm{keV}$ which is typical for a SEM. Considering the 
advantages and disadvantages, we chose a special designed STM for our investigation of graphene growth.

\begin{tabular}{|c|c|c|c|c|}
\hline & $\begin{array}{l}\text { Spatial resolution at } \\
\text { high temperature * }\end{array}$ & $\begin{array}{l}\text { Maximum field of } \\
\text { view }\end{array}$ & Sample temperature & $\begin{array}{l}\text { Maximum vacuum } \\
\text { pressure }\end{array}$ \\
\hline STM & $1 \mathrm{~nm}$ & $3 \mu \mathrm{m}$ & $\sim 1200 \mathrm{~K}$ & $1 \times 10^{-3} \mathrm{mbar}$ \\
\hline LEEM & $\begin{array}{l}2 \mathrm{~nm} \text { at room } \\
\text { temperature }\end{array}$ & $75 \mu \mathrm{m}$ & $\sim 1200 \mathrm{~K}$ & $1 \times 10^{-6} \mathrm{mbar}$ \\
\hline SEM & $\begin{array}{l}2 \mathrm{~nm} \text { at room } \\
\text { temperature }\end{array}$ & $4 \mathrm{~mm}$ & $\sim 1200 \mathrm{~K}$ & $1 \times 10^{-4} \mathrm{mbar}$ \\
\hline
\end{tabular}

* There are some debates in the definition of resolution. Only general numbers are list here.

Table 1. Comparison of microcopies that can be used at the conditions for graphene growth on metals. The specifications of each microscopy are very dependent on the specific design. E.g. differential pumping stages might increase the maximum vacuum pressure.

The STM we used had been optimized for (fast) scanning at high sample temperatures and also during substantial temperature variations [48, 49]. Like most other STMs, our VariableTemperature STM (VT-STM) is using a piezoelectric tube, in our case with a length of $12 \mathrm{~mm}$, to scan regions up to typically $3 \times 3 \mu \mathrm{m}^{2}$, with sub-nanometer resolution. The macroscopic dimensions of the piezo element, the sample and other components in the mechanical path between the tip and the sample make that even modest temperature variations can cause a significant drift in an STM image. More dramatically, when the component of this drift in the $\mathrm{z}$-direction, along the tip axis, were to exceed the control range of the piezo element, this would lead to a situation where either the surface drifts out of range or a crash of the tip into the sample surface occurs. For these reasons, most STMs are used either at room temperature or at a constant, low or (modestly) elevated temperature, at which the sample and microscope are first allowed to equilibrate for a long time, typically several hours, prior to the coarse tipsample approach and imaging. The STM we were using could compensate for thermal drifts by its special design [48]. With this design, neither the expansion of the piezo element and other components of the microscope nor that of the sample and sample holder can give rise to a significant tip-sample distance variation. In practice, experiments have been performed without the need for any mechanical adjustments in the tip height, while the sample temperature was being changed from $300 \mathrm{~K}$ to approximately $1200 \mathrm{~K}$.

In addition to the drift-minimizing mechanical design, the STM electronics have been optimized to scan fast. It has been demonstrated that the STM can image surfaces at video rate (24 frames per second) [49] (A commercial version of this instrument is marketed by Leiden Probe Microscopy BV, www.leidenprobemicroscopy.com). Because of the critical experimental conditions, in particular the high temperatures during deposition, video-rate scanning is very difficult. Therefore, the high-speed capabilities of the VT-STM have not been exploited directly in the work described in this chapter. On the other hand, the low noise level that the high- 
speed electronics has at low frequencies and the modular design of the electronics provided stable scanning conditions plus possibilities for customization of the scans, which played an important role in this work.

\section{Experimental detail}

Our special-purpose, VT-STM [48, 49], enables us to follow the reaction and growth of graphene in situ. Ethylene decomposition on $\mathrm{Rh}(111)$ is taken as a generic example of carbon deposition on metals. The interaction between carbon and Rh can lead to carbide formation [50], carbon dissolution [51], and graphene formation [29]. These are also the typical 'products' that can be formed on other metals. Actually, carbon is the main contaminant in Rh crystals.

The complexity of the carbon-on-Rh(111) system makes it a good example for demonstrating, in general, the interaction between carbon and metals. The 3-fold (111) orientation was chosen, to act as a template for graphene crystallization. In addition, graphene forms an easily recognizable moiré pattern on $\mathrm{Rh}(111)$. A simple calculation shows that the moiré pattern 'magnifies' the misfit defects between the lattice of the graphene layer and that of the Rh substrate. For example, 1 degree of actual graphene rotation with respect to the Rh lattice results in a 10 degree apparent rotation in the moiré pattern. In this way, atomic information about graphene can be achieved even without actually achieving atomic resolution in STM.

All measurements in this chapter were performed with a STM-setup which consisted of a single ultrahigh vacuum (UHV) chamber, equipped with a scanning tunneling microscope (STM), a low-energy electron diffraction (LEED) apparatus, an Auger electron spectroscopy (AES) instrument, and a quadrupole mass spectrometer (QMS), for analysis purposes. The base pressure of the vacuum system was $1.5 \times 10^{-11}$ mbar. During measurements, the vacuum level was kept below $2 \times 10^{-10} \mathrm{mbar}$. To achieve and maintain this vacuum, a single 170 liter/second magnetically levitated turbo-molecular pump, a 410 liter/second ion getter pump, and a titanium sublimation pump, integrated into a cold trap, were connected to the UHV chamber. Also, the entire vacuum system was extensively degassed by a bake-out procedure up to 450 $\mathrm{K}$ and the sample holder and STM components were further degassed by prolonged annealing of the Rh sample to $800 \mathrm{~K}$.

The Rh sample was cleaned by cycles of $\mathrm{Ar}^{+}$ion sputtering, followed by flash annealing to 1300 $\mathrm{K}$ and then exposure of the sample to 2 to $3 \times 10^{-7}$ mbar of $\mathrm{O}_{2}$ for 1 to 2 hours at temperatures of 700 to $800 \mathrm{~K}$. This latter step proved necessary in order to remove $\mathrm{C}$ surface contamination emanating from the bulk. Residual $\mathrm{O}$ was removed from the $\mathrm{Rh}$ surface by flashing the sample to $1000 \mathrm{~K}$. After this cycle had been repeated at least 10 times, no C contamination could be detected by Auger Electron Spectroscopy, and the density of visible impurities in the STM images were typically below $1 / \mu \mathrm{m}^{2}$. The dosage of ethylene gas, from ALDRICH ${ }^{\mathrm{TM}}$, with a purity of $99.99 \%$, was through a precision leak valve. 


\section{A universal description of graphene formation on metals}

In this section, we introduce a new physical picture (Figure 1), which descripts the general principles of graphene and carbide formation on metal surfaces. It is based on the combination of our direct STM observations and the considerations published by McCarty et al. [12]. In Figure 1, the black line represents the density of carbon adatoms on the Rh surface, dictated by equilibrium with carbon dissolved in the bulk. The shape and position of this line is determined by the carbon concentration in the substrate, and by the dissolution energy of carbon [12]. The solid red line represents the carbon adatom density derived from equilibrium with the graphene islands. The point where the black line crosses the solid red line gives the dissolution temperature of graphene. We now add the solid blue line, which represents the carbon adatom density which would establish equilibrium with the carbide islands. The positions of the solid red and blue lines are determined by the edge energies of graphene and carbide. The lower position of the line means the edge energy is lower and the island is more stable. The dashed red and blue lines are the supersaturation necessary for observable nucleation of graphene and carbide. The dashed lines are not only determined by the edge energies of graphene and carbide, but also by the critical nucleation size of each product. So it is not necessary that the dashed blue line is higher than the dashed red line, just because the solid blue line is higher than the solid red line. In fact, our experiment shows the dashed red line is higher than the dashed blue one, while the solid blue line is higher than the solid red line. In the following sections, we will experimentally determine the relative position of each line in this figure. Guided by this figure, we show several paths for improving the quality of graphene.

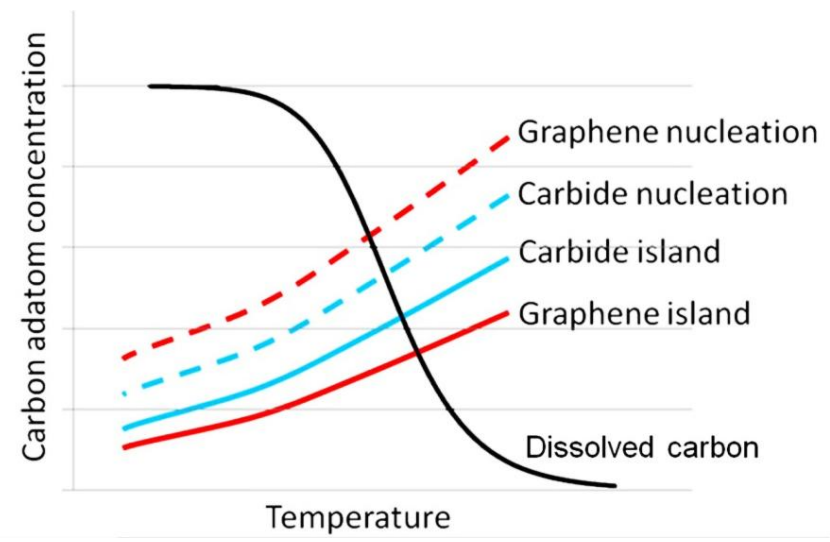

Figure 1. Schematic diagram, showing the temperature dependence of various equilibrium adatom concentrations of carbon on the metal surface. The black line is the carbon adatom concentration, which is in equilibrium with carbon in the bulk. The solid red line is the adatom concentration for equilibrium with graphene islands (after [12]); the solid blue line is the adatom concentration for equilibrium with carbide islands; the locations where the solid lines cross the black line are the dissolution temperatures for graphene and carbide; the dashed red and blue lines indicate the supersaturation levels required for nucleation of graphene and carbide, respectively, on a clean metal surface. 


\section{The temperature dependence of graphene and carbide formation}

In order to obtain a first impression of the temperature dependence of graphene formation, we exposed the Rh surface to a high dose of ethylene at room temperature and slowly heated up the sample, while the surface was monitored continuously with STM. The initial, saturated layer of ethylene was obtained by exposing the freshly cleaned Rh(111) surface [51] in the UHV chamber to $3 \times 10^{-5}$ mbar s of ethylene gas at room temperature. The result of this exposure is a pronounced, atomic-scale roughness, decorating the entire surface (Figure 2A), that we associate with the disordered overlayer of ethylene molecules. During the initial stages of the temperature ramp, clusters formed without any preference for specific edge orientations (Figure 2B). These clusters grew in size when the temperature was increased. Even though we have directly observed the motion and coalescence of the clusters, the drifting during temperature changes makes is difficult to conclude that this was the sole ripening mechanism. Previous research showed ethylene decomposition on $\mathrm{Rh}$ to proceed in various stages at different temperatures up to $\sim 800 \mathrm{~K}[29,52]$. Based on this, we interpret the irregular structures in Figure $2 \mathrm{~B}$ to be clusters of carbon or $\mathrm{CH}_{\mathrm{x}}$. At around $870 \mathrm{~K}$, some islands were observed with a hexagonal shape, and the characteristic moiré pattern inside, indicating that, at this temperature, graphene had already been formed, and that the domains were large enough to appear as moiré patterns. The restructuring of the overlayer into graphene necessarily should start with small domains, smaller than one unit of the moiré pattern $(2 \times 12 \times 12$ carbon atoms). Ripening processes make some domains grow at the expense of others, to become larger than this size. Because any ripening process requires the relocation of many carbon atoms, this ripening should be relatively slow [53]. The combination of this slow ripening with the fast temperature ramp up to $870 \mathrm{~K}$ of $0.2 \mathrm{~K} / \mathrm{s}$, implies that the moiré pattern observed at $870 \mathrm{~K}$ is a sign that graphene formation had started already at a temperature below $870 \mathrm{~K}$. Indeed, Figure $2 \mathrm{C}$ shows our lowest-temperature observation of a moiré pattern, at $808 \mathrm{~K}$, (temperature ramp rate was $0.05 \mathrm{~K} / \mathrm{s}$ ) close to the temperature range of 700-800 K, necessary for complete decomposition of ethylene $[29,52]$. Up to $969 \mathrm{~K}$, the ripening of the graphene islands continued, making the islands larger, more compact and more hexagonal (Figure 2D). Also, the orientations of different domains became similar. Nevertheless, we still observed more than a single moiré pattern at this high temperature, which indicates that several overlayer orientations may have similarly low energies.

In a separate experiment we found that the graphene overlayer dissolved into the Rh substrate, starting at a temperature of $1050 \mathrm{~K}$. The information obtained from these heating experiments can be cast in the form of the upper temperature bar in Figure 2E, displaying that, from $808 \mathrm{~K}$ to $1053 \mathrm{~K}$, graphene can be formed on a Rh surface. This $250 \mathrm{~K}$ temperature range for stable graphene on $\mathrm{Rh}$ is much wider than the $50 \mathrm{~K}$ window that has been reported previously [24]. This process, combining adsorption of ethylene at room temperature with a subsequent temperature ramp, also yields a lower graphene formation temperature than the temperature of $\sim 1100 \mathrm{~K}$, reported in case ethylene was dosed directly at high temperature [54].

From this experiment, we can conclude that graphene is more stable than carbide and that in Figure 1 the solid red line should be lower than the blue one. In this experiment, clustering of 
$\mathrm{CH}_{\mathrm{x}}$ must make the surface carbon concentration locally very high, beyond that shown by the dashed blue and red lines. Under such conditions, both carbide and graphene nuclei can be formed. With time, the carbide islands disappear and the graphene islands become larger, since they are energetically more favourable. This is also why the carbide may form first on metal surfaces, but after long periods of annealing (e.g., 400 minutes) at high temperature, it can transform to graphene [55].

Another experiment was designed to determine the relative position of dashed red and blue lines in Figure 1. In the experiment above, the carbon adatom density in $\mathrm{CH}_{\mathrm{x}}$ is high enough for the nucleation of both graphene and carbide; the two nucleation process cannot be distinguished. To discriminate, we fixed the temperature of a clean Rh sample at $830 \mathrm{~K}$, which is suitable for the formation of graphene and carbide, then we started adding the ethylene starting from zero. On the clean Rh surface, the carbon adatom density was increasing from almost zero, while the pressure of ethylene was being increased. Because there was no nucleus of graphene or carbide on the surface, carbon would form the product (graphene or carbide) which requires the lower nucleation carbon adatom density, during the increasing of the density of carbon adatoms. With STM it was seen that an overlayer formed, starting from the $\mathrm{Rh}$ step edges on the surface, and proceeding until it covered the entire surface. As can be seen in Figure 3A, this overlayer had a structure that was different from that of graphene. After the entire surface had been covered by this layer, we heated the sample to higher temperatures, while monitoring the structure with the STM. It was found that the overlayer did not transform into the graphene structure even up to the temperature of $1016 \mathrm{~K}$, at which it disappeared. The stability of the structure indicates that it did not consist of $\mathrm{CHx}$ clusters, which would otherwise have transformed into graphene at and above $808 \mathrm{~K}$. We interpret the disappearance again as the dissolution of the deposited material into the $\mathrm{Rh}$ substrate. The fact that the dissolution temperature differs significantly from the value of $1050 \mathrm{~K}$, mentioned above for graphene, indicates that in this case the overlayer was not graphene. AES (Figure 3B) on this layer shows that the KLL of carbon peak had shifted from $272 \mathrm{eV}$ to $275 \mathrm{eV}$ and the MNN peak of Rh had also undergone a change in shape and position. Both shifts indicate the formation of a new compound [56]. We suggest that this is a rhodium carbide overlayer. Comparing this AES spectrum with a reference spectrum taken on a one-monolayer graphene-covered Rh surface (Figure 3B), we see that the C-to-Rh peak ratios for these two cases were similar. Assuming that the Rh peak in the AES spectrum of graphene-covered Rh corresponds to effectively one atomic layer of $\mathrm{Rh}$, we find that the $\mathrm{C}$-to-Rh ratio in the carbide must have been in the order of 2:1. In Figure 2E, in analogy to the temperature bar for a graphene overlayer, the lower bar shows the growth and stability regime of a carbide layer on $\mathrm{Rh}(111)$. The fact that carbide was formed first in this experiment means that the require carbon adatom density for nucleation of graphene is higher than that for carbide, which makes the position of the dashed red line higher than dashed blue line in Figure 1. From these two experiments, we can determine the relative position of the lines in Figure 1. It also naturally explains why a lower dissolving temperature was measured for carbide. That is simple because that the solid blue line crosses the black line at a lower temperature than the red one. 


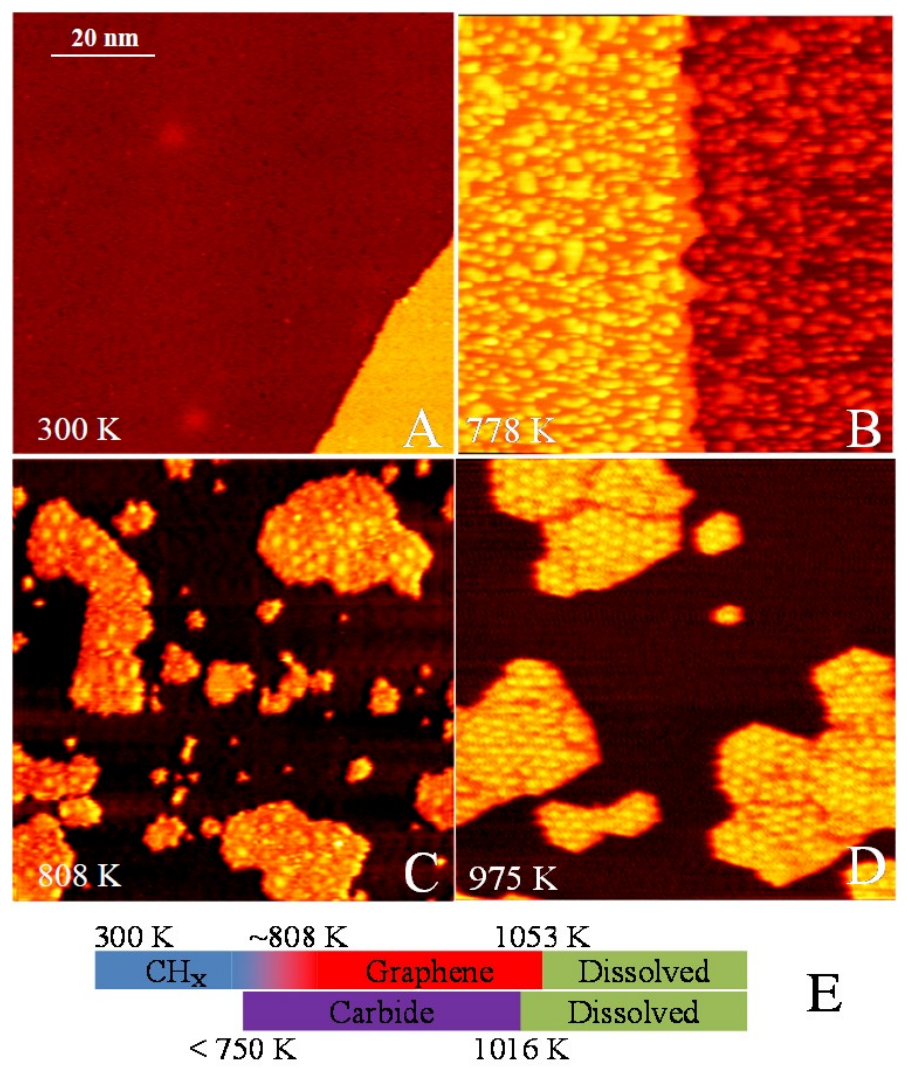

Figure 2. STM images measured during continuous heating from $300 \mathrm{~K}$ to $\sim 1000 \mathrm{~K}$, after room temperature ethylene deposition. Panels A, B and D are from one series of experiments. (A) The Rh(111) surface directly after exposure to $3 x$ $10^{-5}$ mbar s of ethylene at room temperature. A mono-atomic step on the Rh surface crosses the image. The saturated ethylene adsorbed layer causes the rough appearance of the upper and lower terraces. (B) At $778 \mathrm{~K}$, the overlayer organized into irregular clusters, but no moiré pattern was found at this temperature. (C) Starting from $4 \times 10^{-7} \mathrm{mbar} \mathrm{s}$ ethylene exposure at room temperature, this image shows the lowest temperature where the moiré pattern of graphene was found. (D) At $975 \mathrm{~K}$, the graphene had ripened into larger islands, with similar orientations. (E) Temperature ranges for the observed stability of graphene and carbide on $\mathrm{Rh}(111)$. The STM images all have a size of $85 \mathrm{~nm} \times$ $85 \mathrm{~nm}$. They have been taken at sample voltages of $\mathrm{V}_{\mathrm{b}}=0.05 \mathrm{~V}, 1.16 \mathrm{~V}, 1.43 \mathrm{~V}$, and $-1.84 \mathrm{~V}$ for panels $\mathrm{A}, \mathrm{B}, \mathrm{C}$ and $\mathrm{D}$, respectively, and at a tunnel current of $\mathrm{I}_{\mathrm{t}}=0.05 \mathrm{nA}$.

\section{Towards the optimal growth recipe}

\subsection{Direct carbon deposition at high temperature}

To reduce the nucleation density of graphene therefore reduce the defect lines between different nuclei, dosing carbon at a high sample temperature is an obvious solution, since the high temperature largely enhances the diffusion of carbon adatoms. However, the experiment 

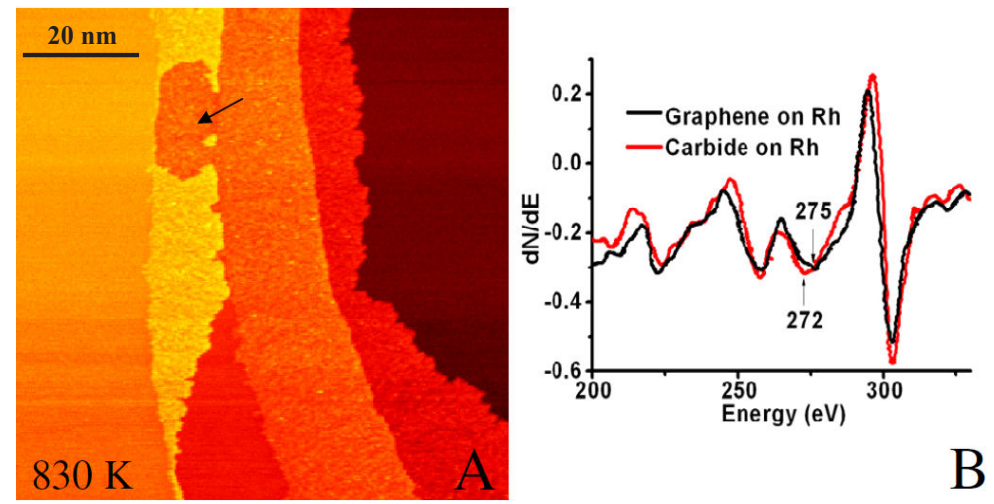

Figure 3. Carbide formation on Rh surface by direct ethylene dosing on a clean Rh. (A) An STM image measured at $830 \mathrm{~K}$, during deposition of ethylene. We interpret the rough layer that started to grow from the Rh steps as a carbide film (see text). Before nucleation of the film, the Rh steps were modified, as is indicated by the arrows. Nucleation started when the ethylene pressure reached $4.4 \times 10^{-8} \mathrm{mbar}$. (B) Comparison between two Auger electron spectra of $\mathrm{Rh}(111)$; red: with a single-monolayer carbide covered Rh, formed by ethylene deposition at 750K, and black: with single-layer graphene. The size of the STM image is $85 \mathrm{~nm} \times 85 \mathrm{~nm}$. It has been taken at sample voltages of $V_{b}=2.79$ $\mathrm{V}$, and at a tunnel current of $\mathrm{I}_{\mathrm{t}}=0.05 \mathrm{nA}$.

above shows that carbide will be formed first on the metal surface before graphene is formed. Interestingly, there is a narrow temperature window, between $1016 \mathrm{~K}$ and $1053 \mathrm{~K}$, in which a carbide layer is no longer stable, but a graphene monolayer is. In order to explore this window, we held the Rh at $1035 \mathrm{~K}$, while dosing with ethylene. Indeed, a graphene layer was observed to form at this temperature. It covered the entire surface without a trace of carbide (Figure $4 \mathrm{~B})$. However, the moiré pattern of the graphene overlayer became distorted during the slow cooling down, after the deposition, as shown in Figure 4C. The low-energy electron diffraction (LEED) pattern that was taken afterwards at room temperature showed a weak $\mathrm{Rh}(111)$ pattern on a strong, diffuse background (inset Figure 4D). We attribute the deterioration of the overlayer during the cooling down to segregation of carbon. It is known that carbon, which dissolves into the near-surface region of the $\mathrm{Rh}$ at the growth temperature, can segregate back to the surface when the temperature is decreased [51]. This segregation could lead to the nucleation of islands of a second graphene or carbide layer, between the original graphene layer and the Rh substrate, which will locally push up the graphene overlayer and distort it.

One may augur that the distortion of graphene moiré pattern might be caused by the different thermal expansion coefficient between graphene and the Rh substrate (in this temperature regime graphene has a negative expansion coefficient $[57,58])$. This effect can be excluded, since other experiments, in which the role of segregated carbon could be minimized (Figure 5), showed that the quality of the graphene was not affected solely by large changes in temperature.

Now we turn to the role of dissolved carbon atoms. When we exposed a clean $\mathrm{Rh}(111)$ substrate directly to ethylene at $1035 \mathrm{~K}$, several dark spots appeared in the STM images, before the graphene was formed (Figure $4 \mathrm{~A}$ ). Graphene formation only started when the ethylene 
pressure reached $3.5 \times 10^{-7}$ mbar, which we associate with the dashed red line in Figure 1 . In the framework of Figure 1, it is natural to ascribe the dark spots in Figure 4A to dissolved carbon atoms, because the supersaturation required for graphene nucleation is high enough for noticeable carbon dissolution, just before nucleation. This dissolution leads to a relatively high concentration of carbon atoms in the substrate, close to the surface. Since the solubility of carbon in the Rh is heavily dependent on temperature, there is a strong driving force for the dissolved carbon atoms to segregate during cooling and accumulate between the Rh substrate and the graphene overlayer. The resulting accumulation layer distorted the regular graphene moiré patterns, as shown in Figure 4C. Other STM measurements of ours also showed that it was possible to re-dissolve these extra carbon atoms by heating the sample to the growth temperature. We speculate that this is only possible as long as the density of carbon in the accumulation layer is not sufficient for the formation of a second layer of graphene. In the latter case, we should have observed the top graphene layer dissolve together with the accumulated (graphene) layer. Note, that the high concentration of dissolved carbon atoms led to a higher dissolution temperature (1118 K) of graphene for this sample, in agreement with our expectation from Figure 1.

Figure 1 also shows that in order to have less carbon dissolved during the direct deposition; one should use a higher pressure. The argument for this counterintuitive approach is that by increasing the pressure the nucleation rate increases super-linearly, whereas the rate of dissolution will simply be proportional to the pressure. Following this idea, we have been able to improve the quality of the graphene overlayer on $\mathrm{Rh}$ as is demonstrated in Figure 4D. The temperature of the clean $\mathrm{Rh}$ sample was first raised to $1028 \mathrm{~K}$, after which the ethylene pressure was quickly increased to a high value of $3.9 \times 10^{-6} \mathrm{mbar}$. The graphene layer that formed under these conditions covered the whole surface with a much lower defect density than the graphene structure shown in Figure 4B. However, in spite of the high ethylene pressure used here, the moiré pattern still distorted due to segregated carbon when the sample was cooled to room temperature. The combination of the high solubility of carbon in Rh and the macroscopic thickness of our substrate $(1 \mathrm{~mm})$ made it practically impossible to completely avoid carbon dissolution at high temperatures and segregation of dissolved carbon during the cool-down.

One of the recipes reported for a single monolayer of graphene on $\mathrm{Rh}(111)$ is to expose the clean rhodium surface for 3 minutes to $2 \times 10^{-7}$ mbar ethylene at an even higher temperature of $1100 \mathrm{~K}$ [54]. We have monitored this recipe with our STM at this high temperature, but graphene was not formed under these conditions and also not with longer exploring times up to 10 minutes. Instead, our images show that in this case a graphene layer was formed only after the exposure, during cooling of the sample, when the temperature had reduced to 1000 $\mathrm{K}$, showing that, in this recipe, the graphene film is assembled completely from segregated carbon. The STM images at room temperature did not show distortions, in accordance with the images in Wang et al. [54]. We attribute this absence of distortions to a nearly complete consumption of the dissolved carbon in the near-surface region by the graphene monolayer. The difficulty in this procedure is to precisely control the coverage, i.e., the total amount of segregated carbon. It is relatively easy to obtain large areas covered by graphene suitable for STM imaging or for a local spectroscopic measurement. But it is very difficult to reach full 

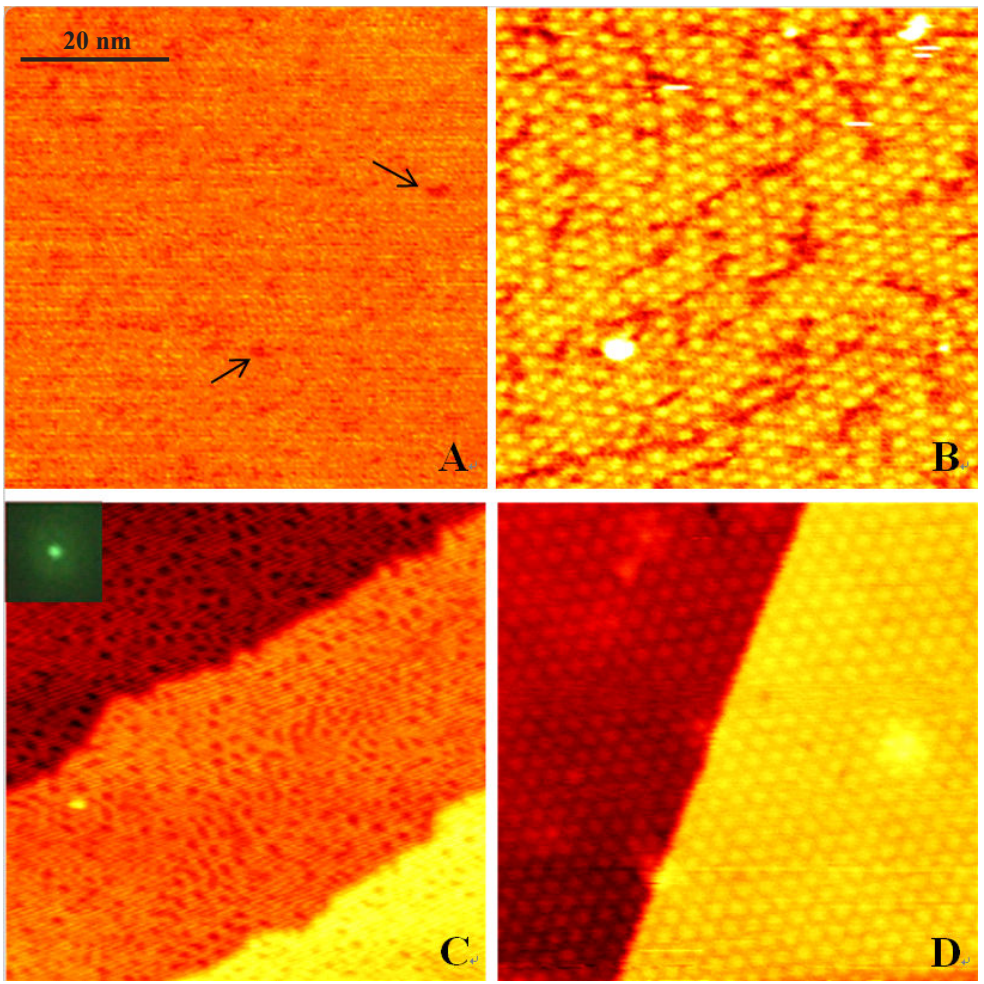

Figure 4. Graphene formation by direct ethylene dosing at high temperature. (A) STM image before the whole surface was covered by graphene. Some dark points (indicated by the arrows), which can be caused by dissolved carbon, are visible. The temperature of the sample was $1035 \mathrm{~K}$ and the pressure of ethylene was $3.5 \times 10^{-7} \mathrm{mbar}$. (B) A STM image of graphene-covered Rh, which was achieved after panel A. The image was taken at the growth temperature. The 'defect' lines in the image were formed already before the graphene overlayer nucleated. We associate these lines with dissolved carbon atoms. (C) STM image of the sample of (B), after it was cooled to $340 \mathrm{~K}$. The moiré pattern of the graphene was deformed. The inset in (C) shows one integer-order Rh LEED spot (same view as in inset of Figure 5B) and the near absence of superstructure spots around it, recorded at room temperature. (D) STM image of graphene-covered Rh, which was achieved by direct ethylene deposition and imaging at $1028 \mathrm{~K}$. The pressure of ethylene was quickly raised to $3.9 \times 10^{-6}$ mbar. The STM images all have a size of $65 \mathrm{~nm} \times 65 \mathrm{~nm}$. They have been taken at sample voltages of $V_{b}=2.5 \mathrm{~V}, 2.5 \mathrm{~V}, 3.7 \mathrm{~V}$, and $4.8 \mathrm{~V}$ for panels $A$ to $\mathrm{D}$, respectively, and at a tunnel current of $\mathrm{I}_{\mathrm{t}}=0.05$ nA.

coverage of the surface with graphene of precisely one layer thickness, i.e., without any excess carbon.

\subsection{Graphene formation on a seeded surface}

The above experiments demonstrated that the temperature window for producing graphene by direct deposition is quite limited, and graphene formed in this temperature window distorts when the substrate is subsequently cooled to room temperature. On the other hand, the first experiment, which started with deposition at room temperature, showed a wider temperature 
window for graphene formation (upper bar in Figure 2E), starting below and ending above the carbide window (lower bar in Figure 2E). The differences between the two experiments in Figure 2 and Figure 3 suggest that the choice which the growing overlayer had to make between carbide and graphene, in the temperature interval from $800 \mathrm{~K}$ to $1016 \mathrm{~K}$, is fully determined by the structure of the initial overlayer nuclei; in other words, graphene patches will continue to grow as graphene, whereas carbide patches will continue to grow as carbide. This interpretation of the experiments also coincides with the theory described in Figure 1. If the carbon adatom density and the temperature is set between the solid lines and dashed lines and the surface is already partially covered by graphene/carbide, carbon adatoms can only contribute the growth of the existing graphene/carbide islands without forming new nuclei. In turn, this suggests a refinement of our approach, namely to separate the stage of nucleation from that of further growth. For example, one could start with the graphene-seeded Rh surface from the first experiment, and expose that to further ethylene deposition under different conditions, in order to obtain full coverage by graphene. One may expect nucleation and growth of the graphene overlayer to follow the well-known rule, in which the nucleation density is determined by $\mathrm{F} / \mathrm{D}$, where $\mathrm{F}$ is the flux of arriving atoms and $\mathrm{D}$ is their surface diffusion coefficient [59]. If the deposition is carried out very slowly, so that the value determined by F/D is smaller than the existing seed density on the surface, the newly arriving carbon atoms will all be incorporated onto the edges of growing graphene islands, which prevents them from forming new nuclei [60]. The presence of the graphene seeds on the surface should have the additional effect that newly arriving carbon atoms have a strongly reduced probability to dissolve into the $\mathrm{Rh}$, again because they will be incorporated in the graphene overlayer on a much shorter timescale, due to the difference in the rates of diffusion of the carbon atoms into the bulk and over the surface, which should be expected to be significant. On a non-seeded surface, dissolution has to compete only with the rather rare processes of nucleation of either graphene or of carbide. How 'difficult' graphene nucleation is on a hot Rh surface is evidenced by our findings in a separate experiment, on a clean Rh surface: at $1035 \mathrm{~K}$, an ethylene pressure of to $3.5 \times 10^{-7}$ mbar was required to observe the first graphene nuclei in our STM images.

The suggested approach, of ethylene deposition at high temperatures onto a Rh surface that has been pre-seeded with graphene by low-temperature deposition, was successful. Figure 5 shows the result obtained at a growth temperature of $975 \mathrm{~K}$; even more informative is the corresponding STM movie, which can be found online. Starting with the end situation of the first experiment (Figure 2D), ethylene was deposited, at a pressure of $3 \times 10^{-9} \mathrm{mbar}$, which was increased at the end of the procedure to $1 \times 10^{-8} \mathrm{mbar}$, simply to accelerate the process. The newly arrived carbon atoms continued with the structure and orientation of the graphene that was already present, until the entire surface was covered with graphene. When a new kink formed at a concave corner between two differently oriented domains, it showed a preference for following the graphene domain which had the same, or a similar orientation, as the Rh crystal. In this way, the metal substrate guided the orientation of the graphene layer, and the density of domain boundaries became lower than that expected from the initial configuration of the graphene seeds. After this sample had been cooled down to room temperature, neither STM nor LEED (insert of Figure 5B) showed a deformation of the moiré pattern. The LEED pattern showed that the graphene layer had a preferred orientation; otherwise, a ring of 

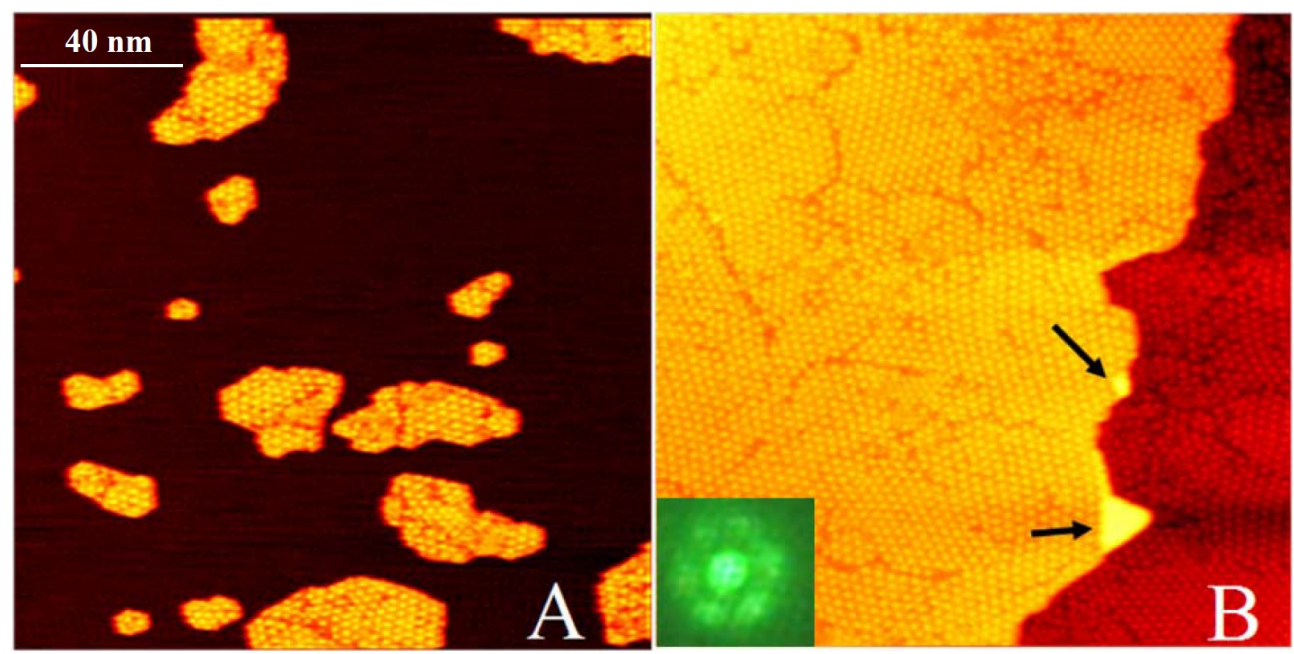

Figure 5. STM images of graphene formation, starting with a seeded surface. (A) The graphene-seeded Rh surface achieved by annealing a pre-deposited sample from room temperature to $975 \mathrm{~K}$. Most of the graphene islands had same orientation. But superstructure domain boundaries can be found within individual islands. (B) Graphene-covered $\mathrm{Rh}$, after ethylene deposition at $975 \mathrm{~K}$ on the seeded surface, at pressures ranging from $3 \times 10^{-9}$ to $1 \times 10^{-8} \mathrm{mbar}$ over a period of 76 minutes. During this procedure, The domain boundary density became $\sim 30 \%$ lower than that in the starting situation. (A). Two Rh double-layer defects are indicated by the arrows. The inset in (B) shows the superstructure spots around one Rh LEED spot. All images are $160 \mathrm{~nm} \times 160 \mathrm{~nm}$ and have been taken at a sample voltage of $V_{b}=$ $-1.84 \mathrm{~V}$ and a tunneling current of $\mathrm{I}_{\mathrm{t}}=0.05 \mathrm{nA}$.

intensity should have been observed around the integer-order maxima from the Rh, rather than the six superstructure spots that are clearly present in the LEED pattern. Although the superstructure spots are not very sharp, the orientation difference between graphene domains is seen to be minor, because the moiré pattern is an amplifier of all variations in position and orientation. The bright regions, indicated by black arrows in Figure 5B, were formed in the final stage of the deposition. They will be explained later.

\subsection{Using segregated carbon to form graphene}

In the previous section, it was shown that the deposition of ethylene can be controlled to form exclusively graphene rather than carbide, and to bring the process to a lower temperature. In this section we show that as an alternative to direct deposition, also the segregation of dissolved carbon atoms could be used in a controlled way, in order to form graphene. By cooling down the sample very slowly, the effective flux of segregated carbon is kept low. These carbon atoms also tend to follow the structure and orientation of the pre-existing seeds; if these have the graphene structure, this procedure results in the growth of graphene. After the segregation has come to an end, the coverage of the graphene overlayer can be brought to completion by a final ethylene exposure. This lower process temperature will be associated with a lower density of carbon dissolved in the substrate and, if it is of any importance, also with a lower 
strain due to the differential thermal expansion between the graphene overlayer and the $\mathrm{Rh}$ substrate $[57,58]$.

This alternative procedure using segregated carbon to form graphene was also successful, as is shown in Figure 6A, where a part of the surface was followed from 862 to $829 \mathrm{~K}$. This is very promising for achieving 2D single crystalline graphene, using dissolved carbon. One difference, relative to deposited carbon, is that the graphene layer also chose the growth direction (Figure 6E) where the Rh step has to retract, to allow further graphene growth. To emphasize that this proceeds against the direction of regular step-flow growth, we refer to this as 'antistep flow'; in some papers it is called 'growth by etching step edges' [61]. In this temperature regime, Rh atoms should be expected to diffuse much faster over the surface than the carbon atoms can in graphene. When sites become temporarily available, as a result of step fluctuations of the Rh, newly arriving carbon atoms may fill up the vacant sites, the strong cohesion in the graphene preventing the Rh step from fluctuating completely back to its original shape and location. This anti-step-flow mechanism resulted in a large number of Rh islands enclosed by graphene. Figure 6C shows one of Rh islands enclosed by graphene, to which more ethylene had been added. The carbon introduced by the ethylene deposition was found to also be accommodated via the anti-step-flow mechanism, instead of forming graphene on top of the enclosed Rh. In this stage, Rh atoms were pushed up and nucleated a higher atomic layer; the graphene stopped growing when the complete enclosed $\mathrm{Rh}$ island had a double-layer height (Figure 6A). The reason that, in Figure 6A, the Rh did not become a double-layer structure, is that, in that case, there was a narrow passage in the graphene layer, through which the $\mathrm{Rh}$ could diffuse away. In separate experiments, in which the Rh was exposed to ethylene at a low temperature of $864 \mathrm{~K}$, the growth of graphene, both at the free edges of graphene islands ('stepflow' growth) and growth against the Rh steps ('anti-step-flow') was observed. At this temperature, the growth rates for these two mechanisms were comparable. At the higher temperatures of the experiments discussed before, e.g. at $975 \mathrm{~K}$, the anti-step-flow growth rate was much lower than the step-flow growth rate. That the mechanism was still active is illustrated by the appearance of double-layer defects in the final stages of the graphene growth, as is indicated by the two arrows in Figure 5B. The temperature dependence of the ratio between the two growth rates can be explained as follows. Assume that decomposition of ethylene or the growth of graphene has a lower energy barrier at the step edges of Rh than on the terraces. At $864 \mathrm{~K}$, the resulting higher decomposition or growth rate of ethylene at the Rh step makes the amount of carbon atoms available for anti-step-flow higher than that for stepflow growth. At $975 \mathrm{~K}$, all ethylene on the Rh terraces rapidly decomposes, which means that the carbon production rate is not limited, at that temperature, by the decomposition, but by the deposition of ethylene. Since the steps represent a much smaller effective area than the terraces, and the anti-step-flow requires an additional process of moving Rh atoms, the ratio between anti-step-flow and step-flow should become low at high temperatures. Nevertheless, anti-step-flow will still occur, and it will lead to Rh double-layer defects. The only way to reduce their density is by further elevating the temperature or by making the average distance between the graphene nuclei larger than the average distance between the steps on the Rh substrate. 

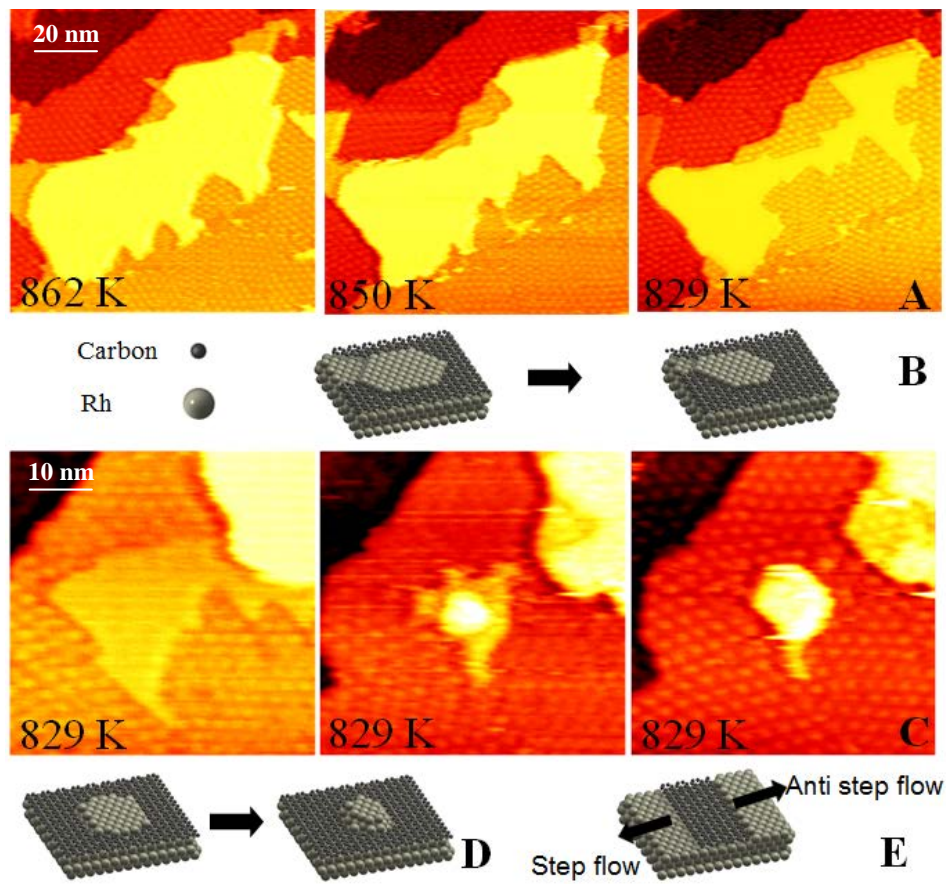

Figure 6. Graphene formation by dissolved carbon and Rh double layer defect formation. (A) STM images of the same area during the cooling down of a Rh sample, partly covered by graphene, after adsorption of ethylene at room temperature and annealing to $977 \mathrm{~K}$. The Rh island indicated by the red arrow is in the same atomic level as the graphene indicated by black arrow. They seem to be in different levels because of the sample voltage setting. The segregated carbon followed the orientation of the existing graphene. Image size: $100 \times 100 \mathrm{~nm}^{2}$. (B) A simple atomic model of the graphene formation by segregated carbon. The Rh island became smaller, because Rh diffused away via the small opening in the graphene layer. (C) Graphene growth, by adding more ethylene while the sample was held at $829 \mathrm{~K}$. These images show how a Rh island, enclosed by graphene, was pushed into a double layer island with half of the original area. Growth of the graphene stopped after the whole island had become a double layer. Image size: $50 \times 50$ $\mathrm{nm}^{2}$. (D) A sketch of the formation of the Rh double layer island. (E) The concept of step flow and anti step flow. In the latter case, Rh atoms have to move to the upper atomic level, in order for the surface to accommodate the additional carbon.

\section{Conclusions}

In this chapter we presented information with both scientific and technical aspects. Technically, the performance of the VT-STM was demonstrated, showing what kinds of extra information can be achieved with this kind of setup. Scientifically, we introduced the schematic 
diagram in Figure 1 which can explain the reported experimental results. We have used insitu measurements to determine the correctness of the picture, and use it to guide the further improvement of graphene quality. This picture can easily be adapted to other CVD procedures on metals. The key elements that may vary from system to system are the stable phases, their formation energies and step energies, the dissolution energy, and the critical nucleation size.

Guided the picture presented, we show three different paths to improve the quality of graphene, namely direct ethylene dosing on clean $\mathrm{Rh}$, first nucleation and growth, and using segregated carbon to form graphene. All of these methods can effectively avoid the formation of carbide. For the method of direct ethylene dosing, the temperature window to avoid carbide formation is quite narrow. The size of the produced graphene domain is quite large. Although the dissolution of carbon cannot be avoided and the segregation of carbon between graphenemetal interface which distorted the moiré pattern of graphene on $\mathrm{Rh}$, the top graphene layer stayed unchanged. This was confirmed by the observation that the moiré pattern became visible again when the sample was heated to the growth temperature. If one wants to produce graphene with larger domains and one does not care about the carbon between the graphene and the metal, it is an easy recipe to follow. In the case of seeding followed by growth, the system is kinetically trapped in a 'graphene-forming' mode. Graphene islands are already present and only a low carbon adatom density is needed to continue the growth. Because of the low ethylene pressure and the rapid incorporation of the deposited carbon into the graphene matrix, the density of adatoms cannot become high enough for nucleation of a carbide (or new graphene islands). Additionally, the dissolved carbon problem is also partially avoided. So all this taken together, the final result is a surface fully covered by graphene. The disadvantage using the seeding method is that the domain density of the graphene is much higher than the one made direct ethylene dosing. The quality of the graphene overlayer is limited by defects in the form of two different classes of domain boundaries. One class results from the merger of neighboring graphene islands with different orientations. On the atomic scale, this type of domain boundary must involve defects with respect to the hexagonal structure of perfect graphene, for example arrays of pentagons and/or heptagons [13] instead of the normal hexagons. The other class of domain boundaries originates from the merger of islands with precisely the same orientation. Due to the mismatch in lattice spacing between the overlayer and the substrate, there are still 288 translationally inequivalent possibilities to position the graphene on the $\mathrm{Rh}(111)$. This makes the probability for a perfect fit lower than $0.4 \%$, so that most of these mergers will be accompanied by a phase defect line in the moiré pattern. Although such lines may look 'dramatic', we speculate that this phase defect is not leading to dangling bonds, i.e., the defect is present only in the moiré period of the superstructure but not in the topology of the graphene network [13]. An alternative path to reduce the domain boundary density to a value which is even lower than the one made by direct ethylene dosing is to reduce the initial nucleation. Other groups also have noticed the importance of initial nucleation [44,60] and used the two step growth method. However, the nucleation conditions (i.e. precursor pressure and temperature) were normally used for the second step of growth after seeding. In this way, the nucleation density in the final product cannot be reduced lower than the intrinsic nucleation density ruled by the growth condition. We now propose that the growth condition should be controlled between the solid and dashed 
lines. In this case, the carbon adatom density is only high enough to continue the growth of graphene, the intrinsic nucleation density by this adatom density can be very low. If the initial nucleation is also set to be very low, e.g. one on entire metal surface, the nucleation density in the final product can be much lower than the graphene made by direct ethylene dosing. With carful control, one single graphene domain over a macroscopic area is very well possible. Our attempts to use segregated carbon plus further ethylene deposition at low temperature to form a complete, undistorted graphene overlayer were successful: the added graphene follows the graphene phase and orientation of the initially nuclei. This procedure forms a very promising route for achieving 2D single-crystalline graphene. However, in the case of Rh substrate, the low growth temperature results in a high growth rate of graphene in the anti-step-flow direction. This results in many Rh double-layer defects, which form holes in the final graphene overlayer.

How to control the thickness of the graphene, in particular how to avoid the nucleation of the second layer below the first layer, now seems evident: after the (first) graphene layer has been formed on the metal surface, the nucleation of a second layer can be avoided by cooling down the sample very rapidly. Also by choosing a metal with a low solubility for carbon, for example, $\mathrm{Cu}$, nucleation of the second layer can be further suppressed.

The physical picture we have shown here explains also why the reported recipes are sometimes not very reproducible. For example, the carbon adatom density is a function of the bulk carbon concentration. In a non-equilibrium case, it also depends on the time during which the sample was held at elevated temperature, and the rate of increase or decrease of the temperature. These parameters vary from case to case and are often not specified.

The central message of present work is that by following an appropriate reaction path through the diagram in Figure1 one can control the formation of the three carbon phases: graphene, carbide, and dissolved carbon. Recipes for producing high-quality graphene should not only consider temperature and pressure, but also the initial situation, the rate of temperature increase/decrease, and the bulk carbon concentration.

\section{Acknowledgements}

This work is part of the research program of the Foundation for Fundamental Research on Matter (FOM), which is part of the Netherlands Organization for Scientific Research (NWO).

\section{Author details}

Guocai Dong $^{1,2}$, Dirk W. van Baarle ${ }^{1}$ and Joost W. M. Frenken ${ }^{1}$

1 Kamerlingh Onnes Laboratory, Leiden University, Leiden, The Netherlands

2 JiangNan Graphene Research Institute, Changzhou, China 


\section{References}

[1] Wallace, P. R. The Band Theory of Graphite. Physical Review, (1947). , 622.

[2] Geim, A. K, \& Novoselov, K. S. The rise of graphene. Nature Materials, (2007). , 183-191.

[3] Novoselov, K. S, et al. Room-Temperature Quantum Hall Effect in Graphene, (2007). , 1379.

[4] Castro NetoA.H., et al., The electronic properties of graphene. Reviews of Modern Physics, (2009). , 109-162.

[5] Du, X, et al. Fractional quantum Hall effect and insulating phase of Dirac electrons in graphene. Nature, (2009). , 192-195.

[6] Westervelt, R. M. Applied physics- Graphene nanoelectronics. Science, (2008). , 324-325.

[7] Britnell, L, et al. Field-Effect Tunneling Transistor Based on Vertical Graphene Heterostructures. Science, (2012). , 947-950.

[8] Lin, Y, et al. GHz Transistors from Wafer-Scale Epitaxial Graphene. Science, (2010). , 662.

[9] Loginova, E, et al. Factors influencing graphene growth on metal surfaces. New Journal of Physics, (2009). , 063046.

[10] Martoccia, D, et al. Graphene on Ru(0001): A $25 \times 25$ supercell. Physical Review Letters, (2008). , 126102.

[11] Oshima, C, et al. A heteroepitaxial multi-atomic-layer system of graphene and h-BN. Surface Review and Letters, (2000). , 521-525.

[12] Mccarty, K. F, et al. Kinetics and thermodynamics of carbon segregation and graphene growth on $\mathrm{Ru}$ (0001). Carbon, (2009). , 1806-1813.

[13] Coraux, J, et al. Structural coherency of graphene on $\operatorname{Ir}(111)$. Nano Letters, (2008). , 565-570.

[14] Oshima, C, \& Nagashima, A. Ultra-thin epitaxial films of graphite and hexagonal boron nitride on solid surfaces. Journal of Physics-Condensed Matter, (1997). , 1-20.

[15] Kim, K. S, et al. Large-scale pattern growth of graphene films for stretchable transparent electrodes. Nature, (2009). , 706-710.

[16] Sutter, P. W, Flege, J. I, \& Sutter, E. A. Epitaxial graphene on ruthenium. Nature Materials, (2008). , 406-411.

[17] Wintterlin, J, \& Bocquet, M. L. Graphene on metal surfaces. Surface Science, (2009). , 1841-1852. 
[18] Novoselov, K. S, et al. Electric Field Effect in Atomically Thin Carbon Films. Science, (2004). , 666-669.

[19] Lui, C. H, et al. Ultraflat graphene. Nature, (2009). , 339-341.

[20] Emtsev, K. V, et al. Towards wafer-size graphene layers by atmospheric pressure graphitization of silicon carbide. Nature Materials, (2009). , 203-207.

[21] Hass, J, et al. Highly ordered graphene for two dimensional electronics, in Appl. Phys. Lett(2006). AIP. , 143106.

[22] Berger, C, et al. Electronic Confinement and Coherence in Patterned Epitaxial Graphene. Science, (2006). , 1191-1196.

[23] Rut'kovE.V. and N.R. Gall, Role of edge atoms of graphene islands on metals in nucleation, growth, alkali metal intercalation. Physics of the Solid State, (2009). , 1738-1743.

[24] Rut'kovE.V., A.V. Kuz'michev, and N.R. Gall, Carbon interaction with rhodium surface: Adsorption, dissolution, segregation, growth of graphene layers. Physics of the Solid State, (2011). , 1092-1098.

[25] Dai, B, et al. Rational design of a binary metal alloy for chemical vapour deposition growth of uniform single-layer graphene. Nat Commun, (2011). , 522.

[26] Gao, L, et al. Repeated growth and bubbling transfer of graphene with millimetre-size singlecrystal grains using platinum. Nat Commun, (2012). , 699.

[27] Liu, N, et al. Universal Segregation Growth Approach to Wafer-Size Graphene from NonNoble Metals. Nano Letters, (2010). , 297-303.

[28] Pan, Y, et al. Highly Ordered, Millimeter-Scale, Continuous, Single-Crystalline Graphene Monolayer Formed on Ru (0001) (21pg 2777, 2009). Advanced Materials, (2009). , 2739-2739.

[29] Castner, D. G, Sexton, B. A, \& Somorjai, G. A. Leed and Thermal Desorption Studies of Small Molecules (H2,O2,Co,Co2,C2h4C2h2 and C) Chemisorbed on Rhodium (111) and (100) Surfaces. Surface Science, (1978). , 519-540.

[30] Isett, L. C, \& Blakely, J. M. Segregation Isosteres for Carbon at (100) Surface of Nickel. Surface Science, (1976). , 397-414.

[31] Shelton, J. C, Patil, H. R, \& Blakely, J. M. Equilibrium Segregation of Carbon to a Nickel (111) Surface-Surface Phase-Transition. Surface Science, (1974). , 493-520.

[32] Oshima, C, et al. A hetero-epitaxial-double-atomic-layer system of monolayer graphenel monolayer h-BN on Ni(111). Solid State Communications, (2000). , 37-40.

[33] Oshima, C, et al. Hetero-epitaxial double-atomic-layer system of monolayer graphene monolayer $h-B N$ on Ni(111) studied by HREELS. Microbeam Analysis 2000, Proceedings, (2000). , 313-314. 
[34] Yu, Q. K, et al. Graphene segregated on Ni surfaces and transferred to insulators. Applied Physics Letters, (2008). , 113103.

[35] Goriachko, A, \& Over, H. Modern Nanotemplates Based on Graphene and Single Layer h$B N$. Zeitschrift Fur Physikalische Chemie-International Journal of Research in Physical Chemistry \& Chemical Physics, (2009). , 157-168.

[36] Bertoni, G, et al. First-principles calculation of the electronic structure and EELS spectra at the graphene/Ni(111) interface. Physical Review B, (2005). , 075402.

[37] Karpan, V. M, et al. Theoretical prediction of perfect spin filtering at interfaces between close-packed surfaces of Ni or Co and graphite or graphene. Physical Review B, (2008). , 195419.

[38] Sutter, P. EPITAXIAL GRAPHENE How silicon leaves the scene. Nature Materials, (2009). , 171-172.

[39] Reina, A, et al. Large Area, Few-Layer Graphene Films on Arbitrary Substrates by Chemical Vapor Deposition. Nano Letters, (2009). , 30-35.

[40] Li, X, et al. Large-Area Graphene Single Crystals Grown by Low-Pressure Chemical Vapor Deposition of Methane on Copper. Journal of the American Chemical Society, (2011). , 2816-2819.

[41] Liu, W, et al. Synthesis of high-quality monolayer and bilayer graphene on copper using chemical vapor deposition. Carbon, (2011). , 4122-4130.

[42] Li, X. S, et al. Large-Area Synthesis of High-Quality and Uniform Graphene Films on Copper Foils. Science, (2009). , 1312-1314.

[43] Bae, S, et al. Roll-to-roll production of 30-inch graphene films for transparent electrodes. Nat Nano, (2010). , 574-578.

[44] $\mathrm{Yu}, \mathrm{Q}$, et al. Control and characterization of individual grains and grain boundaries in graphene grown by chemical vapour deposition. Nature Materials, (2011). , 443-449.

[45] Gunther, S, et al. Single Terrace Growth of Graphene on a Metal Surface. Nano Letters, (2011). , 1895-1900.

[46] Dong, G. C, et al. Graphene formation on metal surfaces investigated by in-situ scanning tunneling microscopy. New Journal of Physics, (2012). , 053033.

[47] Loginova, E, et al. Evidence for graphene growth by C cluster attachment. New Journal of Physics, (2008). , 093026.

[48] Hoogeman, M. S, et al. Design and performance of a programmable-temperature scanning tunneling microscope. Review of Scientific Instruments, (1998). , 2072-2080.

[49] Rost, M. J, et al. Scanning probe microscopes go video rate and beyond. Review of Scientific Instruments, (2005). , 053710. 
[50] Chen, J. G, et al. Controlling surface reactivities of transition metals by carbide formation. Journal of Molecular Catalysis a-Chemical, (1998). , 285-299.

[51] Delouise, L. A, \& Winograd, N. Carbon-Monoxide Adsorption and Desorption on Rh(111) and $R h$ (331) Surfaces. Surface Science, (1984). , 417-431.

[52] Dubois, L. H, Castner, D. G, \& Somorjai, G. A. The Chemisorption of Acetylene and Ethylene on Rh(111)- a Low-Energy Electron-Diffraction (Leed), High-Resolution Electron-Energy Loss (Els), and Thermal-Desorption Mass-Spectrometry (Tds) Study. Journal of Chemical Physics, (1980). , 5234-5240.

[53] Ibach, H. Physics of Surfaces and Interfaces. (2006). Jülich: Springer-Verlag Berlin Heidelberg. 525., 10

[54] Wang, B, et al. Coupling Epitaxy, Chemical Bonding, and Work Function at the Local Scale in Transition Metal-Supported Graphene. ACS Nano, (2010). , 5773-5782.

[55] Lahiri, J, et al. Graphene Growth on Ni(111) by Transformation of a Surface Carbide. Nano Letters, (2011). , 518-522.

[56] Sinharoy, S, \& Levenson, L. L. The formation and decomposition of nickel carbide in evaporated nickel films on graphite. Thin Solid Films, (1978). , 31-36.

[57] Röhrl, J, et al. Raman spectra of epitaxial graphene on SiC(0001). Applied Physics Letters, (2008). , 201918.

[58] Zakharchenko, K. V, Katsnelson, M. I, \& Fasolino, A. Finite Temperature Lattice Properties of Graphene beyond the Quasiharmonic Approximation. Physical Review Letters, (2009). , 046808.

[59] Venables, J. A. Introduction to Surface and Thin Film Processes. (2001). Cambridge: Cambridge University press.

[60] Van Gastel, R, et al. Selecting a single orientation for millimeter sized graphene sheets. Applied Physics Letters, (2009). , 121901.

[61] Starodub, E, et al. Graphene growth by metal etching on $R u(0001)$. Physical Review B, (2009). , 235422. 